\title{
Two iterative algorithms for solving coupled matrix equations over reflexive and anti-reflexive matrices
}

\author{
MEHDI DEHGHAN ${ }^{1}$ and MASOUD HAJARIAN ${ }^{2 *}$ \\ ${ }^{1}$ Department of Applied Mathematics, Faculty of Mathematics and Computer Science, \\ Amirkabir University of Technology, 424, Hafez Avenue, Tehran 15914, Iran \\ ${ }^{2}$ Department of Mathematics, Faculty of Mathematical Sciences, \\ Shahid Beheshti University, G.C., Tehran 19839, Iran \\ E-mails: mdehghan@aut.ac.ir / mdehghan.aut@gmail.com/m_hajarian@sbu.ac.ir / \\ mhajarian@aut.ac.ir/masoudhajarian@gmail.com
}

Abstract. An $n \times n$ real matrix $P$ is said to be a generalized reflection matrix if $P^{T}=P$ and $P^{2}=I$ (where $P^{T}$ is the transpose of $P$ ). A matrix $A \in \mathbb{R}^{n \times n}$ is said to be a reflexive (antireflexive) matrix with respect to the generalized reflection matrix $P$ if $A=P A P(A=-P A P)$. The reflexive and anti-reflexive matrices have wide applications in many fields. In this article, two iterative algorithms are proposed to solve the coupled matrix equations

$$
\left\{\begin{array}{l}
A_{1} X B_{1}+C_{1} X^{T} D_{1}=M_{1}, \\
A_{2} X B_{2}+C_{2} X^{T} D_{2}=M_{2},
\end{array}\right.
$$

over reflexive and anti-reflexive matrices, respectively. We prove that the first (second) algorithm converges to the reflexive (anti-reflexive) solution of the coupled matrix equations for any initial reflexive (anti-reflexive) matrix. Finally two numerical examples are used to illustrate the efficiency of the proposed algorithms.

Mathematical subject classification: $15 \mathrm{~A} 06,15 \mathrm{~A} 24,65 \mathrm{~F} 15,65 \mathrm{~F} 20$.

Key words: iterative algorithm, matrix equation, reflexive matrix, anti-reflexive matrix.

\#CAM-394/11. Received: 24/VII/11. Accepted: 05/III/12.

*Corresponding author. 


\section{Introduction}

In this paper we use the following notation. Let $\mathbb{R}^{m \times n}$ be the set of all $m \times n$ real matrices. We use $\operatorname{tr}(A), A^{T}, \rho(A), \lambda(A)$ and $\lambda_{\max }(A)$ to denote the trace, the transpose, the spectral radius, the eigenvalue set and the maximum eigenvalue of the matrix $A$ respectively. We denote by $I_{k}$ and $O_{m \times n}$ the $k \times k$ identity matrix and the $m \times n$ zero matrix, respectively. We also write them as $I$ and $O$, respectively, when the dimensions of these matrices are clear. We define an inner product as $\langle A, B\rangle=\operatorname{tr}\left(B^{T} A\right)$, then the norm of a matrix $A$ generated by this inner product is Frobenius norm and is denoted by $\langle A, A\rangle=\|A\|^{2}$.

An $n \times n$ real matrix $P$ is said to be a real generalized reflection matrix if $P^{T}=P$ and $P^{2}=I$. An $n \times n$ real matrix $A$ is said to be a reflexive (anti-reflexive) matrix with respect to the generalized reflection matrix $P$ if $A=P A P(A=-P A P) . \mathbb{R}_{r}^{n \times n}(P)\left(\mathbb{R}_{a}^{n \times n}(P)\right)$ denotes the subspace reflexive (anti-reflexive) matrices with respect to the $n \times n$ generalized reflection matrix $P$. The reflexive and anti-reflexive matrices have practical applications in many areas such as the numerical solution of certain differential equations [1], pattern recognition [6], Markov processes [42], various physical and engineering problems [7] and so on (e.g. [20, 32, 43]). Chen [3] proposed three applications of reflexive and anti-reflexive matrices obtained from the altitude estimation of a level network, an electric network and structural analysis of trusses. The symmetric Toeplitz matrices, an important subclass of the class of symmetric reflexive matrices, appear naturally in digital signal processing applications and other areas [21].

The linear matrix equations, such as $A X B=C, A X B+C X D=E$ and $A X B+C X^{T} D=M$, play an important role in linear system theory therefore a large number of papers have presented several methods for solving these matrix equations $[2,9,15,36]$. Research on solving of linear matrix equations has been actively ongoing for past years. In [5], Dai studied the linear matrix equation

$$
A X B=C,
$$

over symmetric matrix $X$. By using g-inverse, Mitra [38] obtained the common solution of simultaneous matrix equations

$$
\left\{\begin{array}{l}
A_{1} X B_{1}=C_{1}, \\
A_{2} X B_{2}=C_{2} .
\end{array}\right.
$$


Navarra et al. [39] studied a representation of the solution $X$ to the system of matrix equations (1.2). The matrix equation

$$
A X+X^{T} C=B
$$

plays important roles in system theory, such as eigenstructure assignment [29], observer design [4], control of system with input constraint [28], and fault detection [30].

In [40], the necessary and sufficient condition for the existence of the solution to the matrix equation (1.3) and its solution expression was investigated by the generalized inverse matrix. In [37], Cramer's rules for some quaternion matrix equations were obtained within the framework of the theory of the column and row determinants. Kyrchei [35] considered systems of linear quaternionic equations and obtained Cramer's rules for right and left quaternionic systems of linear equations. In $[44,45,46]$, the solutions of the several generalized Sylvester matrix equations were established. In [24], a family of iterative methods for linear systems is presented and a least-squares iterative solution to coupled matrix equations are studied by using the hierarchical identification principle and the star product. In [26], gradient iterative algorithms for solving Sylvester coupled matrix equations and general coupled matrix equations are studied by using the gradient search principle. In [22, 25], Ding and Chen applied a hierarchical identification principle to study solving the Sylvester and Lyapunov matrix equations. Also Ding and Chen [23] proposed a hierarchical gradient iterative algorithm and a hierarchical stochastic gradient algorithm and prove that the parameter estimation errors given by the algorithms converge to zero for any initial values under persistent excitation. In $[8,10,11,12,13,14,17,18]$, Dehghan and Hajarian introduced some efficient iterative methods for solving Sylvester and Lyapunov matrix equations.

In this paper, we introduce two iterative algorithms, respectively, for the finding reflexive and anti-reflexive solutions of the coupled matrix equations

$$
\left\{\begin{array}{l}
A_{1} X B_{1}+C_{1} X^{T} D_{1}=M_{1}, \\
A_{2} X B_{2}+C_{2} X^{T} D_{2}=M_{2},
\end{array}\right.
$$

(including the matrix equations (1.1)-(1.3) as special cases).

The rest of the paper is structured as follows. In Section 2, first we propose two iterative algorithms for solving (1.4) over reflexive and anti-reflexive matrices. 
Then we study the convergence properties of the iterative algorithms. Two examples verify the efficiency of the algorithms in Section 3. Section 4 concludes the paper.

\section{Main results}

In this section, first we give two systems of matrix equations equivalent to (1.4) over reflexive and anti-reflexive matrices, respectively. Then we will propose two efficient iterative algorithms for solving (1.4).

Lemma 2.1. The coupled matrix equations (1.4) have the reflexive solution $X \in \mathbb{R}_{r}^{n \times n}(P)$ if and only if the system of matrix equations

$$
\left\{\begin{array}{l}
A_{1} X B_{1}+C_{1} X^{T} D_{1}=M_{1}, \\
A_{2} X B_{2}+C_{2} X^{T} D_{2}=M_{2}, \\
A_{1} P X P B_{1}+C_{1} P X^{T} P D_{1}=M_{1}, \\
A_{2} P X P B_{2}+C_{2} P X^{T} P D_{2}=M_{2},
\end{array}\right.
$$

is consistent.

Proof. First, we suppose that the coupled matrix equations (1.4) have the reflexive solution $X^{*} \in \mathbb{R}_{r}^{n \times n}(P)$. By using $X^{*}=P X^{*} P$ and $A_{i} X^{*} B_{i}+C_{i} X^{*} T D_{i}=$ $M_{i}$, we have

$$
\begin{aligned}
A_{i} P X^{*} P B_{i}+C_{i} P X^{* T} P D_{i} & =A_{i} X^{*} B_{i}+C_{i}\left(P X^{*} P\right)^{T} D_{i} \\
& =A_{i} X^{*} B_{i}+C_{i} X^{* T} D_{i} \\
& =M_{i},
\end{aligned}
$$

for $i=1,2$. It is follows from (2.2) that the reflexive matrix $X^{*}$ is a solution of the system of matrix equations (2.1).

Conversely assume that the system of matrix equations (2.1) is consistent. Let $X$ be a solution of the system of matrix equations (2.1). Set

$$
\tilde{X}=\frac{X+P X P}{2} .
$$


Therefore $\tilde{X} \in \mathbb{R}_{r}^{n \times n}(P)$ and we can get

$$
\begin{aligned}
A_{i} \tilde{X} B_{i}+C_{i} \tilde{X}^{T} D_{i} & =A_{i}\left(\frac{X+P X P}{2}\right) B_{i}+C_{i}\left(\frac{X+P X P}{2}\right)^{T} D_{i} \\
& =\frac{A_{i} X B_{i}+A_{i} P X P B_{i}}{2}+\frac{C_{i} X^{T} D_{i}+C_{i} P X^{T} P D_{i}}{2} \\
& =\frac{A_{i} X B_{i}+C_{i} X^{T} D_{i}}{2}+\frac{A_{i} P X P B_{i}+C_{i} P X^{T} P D_{i}}{2} \\
& =\frac{M_{i}+M_{i}}{2} \\
& =M_{i},
\end{aligned}
$$

for $i=1,2$. Hence $\tilde{X}$ is a reflexive solution of the coupled matrix equations (1.4). The proof is completed.

Similarly to the above lemma, we can obtain the following lemma.

Lemma 2.2. The coupled matrix equations (1.4) have the anti-reflexive solution $X \in \mathbb{R}_{a}^{n \times n}(P)(P \neq I)$ if and only if the system of matrix equations

$$
\left\{\begin{array}{l}
A_{1} X B_{1}+C_{1} X^{T} D_{1}=M_{1}, \\
A_{2} X B_{2}+C_{2} X^{T} D_{2}=M_{2}, \\
-A_{1} P X P B_{1}-C_{1} P X^{T} P D_{1}=M_{1}, \\
-A_{2} P X P B_{2}-C_{2} P X^{T} P D_{2}=M_{2},
\end{array}\right.
$$

is consistent.

According to Theorem 4.3.8 and Corollary 4.3.10 in [33], the systems (2.1) and (2.5), respectively, are equivalent to

$$
\left(\begin{array}{c}
\left(B_{1}^{T} \otimes A_{1}\right)+\left(D_{1}^{T} \otimes C_{1}\right) P(n, n) \\
\left(B_{2}^{T} \otimes A_{2}\right)+\left(D_{2}^{T} \otimes C_{2}\right) P(n, n) \\
\left(B_{1}^{T} P \otimes A_{1} P\right)+\left(D_{1}^{T} P \otimes C_{1} P\right) P(n, n) \\
\left(B_{2}^{T} P \otimes A_{2} P\right)+\left(D_{2}^{T} P \otimes C_{2} P\right) P(n, n)
\end{array}\right)
$$


and

$$
\left(\begin{array}{c}
\left(B_{1}^{T} \otimes A_{1}\right)+\left(D_{1}^{T} \otimes C_{1}\right) P(n, n) \\
\left(B_{2}^{T} \otimes A_{2}\right)+\left(D_{2}^{T} \otimes C_{2}\right) P(n, n) \\
-\left(B_{1}^{T} P \otimes A_{1} P\right)-\left(D_{1}^{T} P \otimes C_{1} P\right) P(n, n) \\
-\left(B_{2}^{T} P \otimes A_{2} P\right)-\left(D_{2}^{T} P \otimes C_{2} P\right) P(n, n)
\end{array}\right)
$$

where $P(n, n)$ is a permutation matrix [33]. Now by using the above results and considering

$$
Z_{1}:=\left(\begin{array}{c}
\left(B_{1}^{T} \otimes A_{1}\right)+\left(D_{1}^{T} \otimes C_{1}\right) P(n, n) \\
\left(B_{2}^{T} \otimes A_{2}\right)+\left(D_{2}^{T} \otimes C_{2}\right) P(n, n) \\
\left(B_{1}^{T} P \otimes A_{1} P\right)+\left(D_{1}^{T} P \otimes C_{1} P\right) P(n, n) \\
\left(B_{2}^{T} P \otimes A_{2} P\right)+\left(D_{2}^{T} P \otimes C_{2} P\right) P(n, n)
\end{array}\right),
$$

and

$$
Z_{2}:=\left(\begin{array}{c}
\left(B_{1}^{T} \otimes A_{1}\right)+\left(D_{1}^{T} \otimes C_{1}\right) P(n, n) \\
\left(B_{2}^{T} \otimes A_{2}\right)+\left(D_{2}^{T} \otimes C_{2}\right) P(n, n) \\
-\left(B_{1}^{T} P \otimes A_{1} P\right)-\left(D_{1}^{T} P \otimes C_{1} P\right) P(n, n) \\
-\left(B_{2}^{T} P \otimes A_{2} P\right)-\left(D_{2}^{T} P \otimes C_{2} P\right) P(n, n)
\end{array}\right),
$$

the following lemmas are well known [31, 33, 34].

Lemma 2.3. The coupled matrix equations (1.4) have a unique reflexive solution with respect to the generalized reflection matrix $P$ if and only if

$$
\operatorname{rank}\left(\left(Z_{1}, \operatorname{vec}\left(M_{1}, M_{2}, M_{1}, M_{2}\right)\right)\right)=\operatorname{rank}\left(Z_{1}\right)
$$

and $Z_{1}$ has a full column rank. In that case, the reflexive solution of (1.4) can be expressed by the following form

$$
\begin{gathered}
X=\frac{X_{1}+P X_{1} P}{2} \text { where } \\
\operatorname{vec}\left(X_{1}\right)=\left(Z_{1}^{T} Z_{1}\right)^{-1} Z_{1}^{T} \operatorname{vec}\left(M_{1}, M_{2}, M_{1}, M_{2}\right),
\end{gathered}
$$


and the homogenous coupled matrix equations

$$
\left\{\begin{array}{l}
A_{1} X B_{1}+C_{1} X^{T} D_{1}=0, \\
A_{2} X B_{2}+C_{2} X^{T} D_{2}=0,
\end{array}\right.
$$

have a unique reflexive solution $X=0$.

Lemma 2.4. The coupled matrix equations (1.4) have a unique anti-reflexive solution with respect to the generalized reflection matrix $P \neq I$ if and only if $\operatorname{rank}\left(\left(Z_{2}, \operatorname{vec}\left(M_{1}, M_{2}, M_{1}, M_{2}\right)\right)\right)=\operatorname{rank}\left(Z_{2}\right)$ and $Z_{2}$ has a full column rank. In that case, the anti-reflexive solution of (1.4) can be expressed by the following form

$$
\begin{gathered}
X=\frac{X_{1}-P X_{1} P}{2} \text { where } \\
\operatorname{vec}\left(X_{1}\right)=\left(Z_{2}^{T} Z_{2}\right)^{-1} Z_{2}^{T} \operatorname{vec}\left(M_{1}, M_{2}, M_{1}, M_{2}\right),
\end{gathered}
$$

and the homogenous coupled matrix equations (2.11) have a unique anti-reflexive solution $X=0$.

If Lemma 2.3 (Lemma 2.4) is applied for finding the reflexive (anti-reflexive) solution of the coupled matrix equations (1.4), we need to take the inverse of the large matrix $Z_{1}^{T} Z_{1}\left(Z_{2}^{T} Z_{2}\right)$. The above method may turn out to be numerically expensive and are not practical for equations of large systems. Our purpose in this paper is to obtain two iterative methods without any inverse for solving the coupled matrix equations (1.4) over reflexive and anti-reflexive matrices. We extend the idea of the Jacobi and the Gauss-Seidel iterations to solve the coupled matrix equations (1.4) over reflexive and anti-reflexive matrices.

Suppose that $A=M-N$ is a splitting of the matrix $A$. The Jacobi and GaussSeidel procedures for solving the linear system $A x=b$ are typical members of a large family of iterations that have the form

$$
M x^{(k+1)}=N x^{(k)}+b,
$$

with $M=D, N=-(L+U)$ for Jacobi and $M=D+L, N=-U$ for Gauss-Seidel [32]. Here by extending the Jacobi and the Gauss-Seidel iterations and by applying the hierarchical identification principle [23, 25], we present two iterative methods for solving the coupled matrix equations (1.4) over reflexive and anti-reflexive matrices. These iterative methods are derived as follows: 


\section{Algorithm 2.1. To solve (1.4) over reflexive matrix $X$ :}

Step 2.1.1. Input matrices $A, C \in \mathbb{R}^{r \times n}, B, D \in \mathbb{R}^{n \times s}$ and $M \in \mathbb{R}^{r \times s}$;

Step 2.1.2. Choose arbitrary $X(1) \in \mathbb{R}_{r}^{n \times n}(P)$ where $P$ is an $n$-by- $n$ arbitrary generalized reflection matrix and a parameter $\omega \in \mathbb{R}^{+}$;

Step 2.1.3. Calculate

$$
\begin{gathered}
R_{i}(1)=M_{i}-A_{i} X(1) B_{i}-C_{i} X(1)^{T} D_{i}, \quad i=1,2 ; \\
k:=1 ;
\end{gathered}
$$

Step 2.1.4. If $\left\|R_{1}(k)\right\|+\left\|R_{2}(k)\right\|=0$, then stop; Else go to step 2.1.5;

Step 2.1.5.

$$
\begin{gathered}
X(k+1)=X(k) \\
+\frac{\omega}{4} \sum_{i=1}^{2}\left(A_{i}^{T} R_{i}(k) B_{i}^{T}+D_{i} R_{i}(k)^{T} C_{i}+P A_{i}^{T} R_{i}(k) B_{i}^{T} P+P D_{i} R_{i}(k)^{T} C_{i} P\right) \\
R_{i}(k+1)=M_{i}-A_{i} X(k+1) B_{i}-C_{i} X(k+1)^{T} D_{i}, \quad i=1,2
\end{gathered}
$$

Step 2.1.6. If $\left\|R_{1}(k+1)\right\|+\left\|R_{2}(k+1)\right\|=0$, then stop; Else, let $k:=k+1$, go to step 2.1.5.

\section{Algorithm 2.2. To solve (1.4) over anti-reflexive matrix $X$ :}

Step 2.2.1. Input matrices $A, C \in \mathbb{R}^{r \times n}, B, D \in \mathbb{R}^{n \times s}$ and $M \in \mathbb{R}^{r \times s}$;

Step 2.2.2. Choose arbitrary $X(1) \in \mathbb{R}_{a}^{n \times n}(P)$ where $P \neq I$ is an $n$-by- $n$ arbitrary generalized reflection matrix and a parameter $\omega \in \mathbb{R}^{+}$;

Step 2.2.3. Calculate

$$
\begin{gathered}
R_{i}(1)=M_{i}-A_{i} X(1) B_{i}-C_{i} X(1)^{T} D_{i}, \quad i=1,2 ; \\
k:=1 ;
\end{gathered}
$$


Step 2.2.4. If $\left\|R_{1}(k)\right\|+\left\|R_{2}(k)\right\|=0$, then stop; Else go to step 2.2.5;

\section{Step 2.2.5.}

$$
\begin{gathered}
X(k+1)=X(k) \\
+\frac{\omega}{4} \sum_{i=1}^{2}\left(A_{i}^{T} R_{i}(k) B_{i}^{T}+D_{i} R_{i}(k)^{T} C_{i}-P A_{i}^{T} R_{i}(k) B_{i}^{T} P-P D_{i} R_{i}(k)^{T} C_{i} P\right) \\
R_{i}(k+1)=M_{i}-A_{i} X(k+1) B_{i}-C_{i} X(k+1)^{T} D_{i}, \quad i=1,2
\end{gathered}
$$

Step 2.2.6. If $\left\|R_{1}(k+1)\right\|+\left\|R_{2}(k+1)\right\|=0$, then stop; Else, let $k:=k+1$, go to step 2.2.5.

Now convergence properties of Algorithms 2.1 and 2.2 are presented.

Theorem 2.1. If the coupled matrix equations (1.4) have a unique reflexive solution $X$, then iterative solution $X(k)$ given by Algorithm 2.1 converges to $X$ for any initial reflexive matrix $X(1)$, if the parameter $\omega$ satisfies the inequality

$$
0<\omega<2\left[\sum_{i=1}^{2}\left(\left\|A_{i}\right\|^{2}\left\|B_{i}\right\|^{2}+\left\|C_{i}\right\|^{2}\left\|D_{i}\right\|^{2}\right)\right]^{-1} \text {. }
$$

Proof. We define the estimation error matrix in the form

$$
\varepsilon(k)=X(k)-X, \quad \text { for } \quad k=1,2, \ldots
$$

By applying (2.15), we can get

$$
R_{i}(k)=-A_{i} \varepsilon(k) B_{i}-C_{i} \varepsilon(k)^{T} D_{i}, \quad \text { for } \quad i=1,2,
$$

Also it is not difficult to obtain

$$
\begin{gathered}
\varepsilon(k+1)=\varepsilon(k) \\
-\frac{\omega}{4} \sum_{i=1}^{2}\left[A_{i}^{T}\left(A_{i} \varepsilon(k) B_{i}+C_{i} \varepsilon(k)^{T} D_{i}\right) B_{i}^{T}+D_{i}\left(A_{i} \varepsilon(k) B_{i}+C_{i} \varepsilon(k)^{T} D_{i}\right)^{T} C_{i}\right. \\
\left.+P A_{i}^{T}\left(A_{i} \varepsilon(k) B_{i}+C_{i} \varepsilon(k)^{T} D_{i}\right) B_{i}^{T} P+P D_{i}\left(A_{i} \varepsilon(k) B_{i}+C_{i} \varepsilon(k)^{T} D_{i}\right)^{T} C_{i} P\right]
\end{gathered}
$$


Now we can write

$$
\begin{aligned}
& \|\varepsilon(k+1)\|^{2}=\operatorname{tr}\left(\varepsilon(k+1)^{T} \varepsilon(k+1)\right) \\
& =\|\varepsilon(k)\|^{2}-\frac{\omega}{2} \operatorname{tr}\left(\varepsilon ( k ) ^ { T } \sum _ { i = 1 } ^ { 2 } \left[A_{i}^{T}\left(A_{i} \varepsilon(k) B_{i}+C_{i} \varepsilon(k)^{T} D_{i}\right) B_{i}^{T}\right.\right. \\
& \left.+D_{i}\left(A_{i} \varepsilon(k) B_{i}+C_{i} \varepsilon(k)^{T} D_{i}\right)^{T} C_{i}\right) \\
& +P A_{i}^{T}\left(A \varepsilon(k) B_{i}+C_{i} \varepsilon(k)^{T} D_{i}\right) B_{i}^{T} P \\
& \left.\left.+P D_{i}\left(A_{i} \varepsilon(k) B_{i}+C_{i} \varepsilon(k)^{T} D_{i}\right)^{T} C_{i} P\right]\right) \\
& +\frac{\omega^{2}}{16} \| \sum_{i=1}^{2}\left[A_{i}^{T}\left(A_{i} \varepsilon(k) B_{i}+C_{i} \varepsilon(k)^{T} D_{i}\right) B_{i}^{T}\right. \\
& +D_{i}\left(A_{i} \varepsilon(k) B_{i}+C_{i} \varepsilon(k)^{T} D_{i}\right)^{T} C_{i} \\
& +P A_{i}^{T}\left(A_{i} \varepsilon(k) B_{i}+C_{i} \varepsilon(k)^{T} D_{i}\right) B_{i}^{T} P \\
& \left.+P D_{i}\left(A_{i} \varepsilon(k) B_{i}+C_{i} \varepsilon(k)^{T} D_{i}\right)^{T} C_{i} P\right] \|^{2} \\
& \leq\|\varepsilon(k)\|^{2}-\frac{\omega}{2} \sum_{i=1}^{2} \operatorname{tr}\left(A_{i} \varepsilon(k) B_{i}\left(A_{i} \varepsilon(k) B_{i}+C_{i} \varepsilon(k)^{T} D_{i}\right)^{T}\right. \\
& +C_{i} \varepsilon(k)^{T} D_{i}\left(A_{i} \varepsilon(k) B_{i}+C_{i} \varepsilon(k)^{T} D_{i}\right)^{T} \\
& +A_{i} P \varepsilon(k) P B_{i}\left(A_{i} \varepsilon(k) B_{i}+C_{i} \varepsilon(k)^{T} D_{i}\right)^{T} \\
& \left.+C_{i} P \varepsilon(k)^{T} P D_{i}\left(A_{i} \varepsilon(k) B_{i}+C_{i} \varepsilon(k)^{T} D_{i}\right)^{T}\right) \\
& +\frac{\omega^{2}}{4}\left\{\left\|\sum_{i=1}^{2} A_{i}^{T}\left(A_{i} \varepsilon(k) B_{i}+C_{i} \varepsilon(k)^{T} D_{i}\right) B_{i}^{T}\right\|^{2}\right. \\
& +\left\|\sum_{i=1}^{2} D_{i}\left(A_{i} \varepsilon(k) B_{i}+C_{i} \varepsilon(k)^{T} D_{i}\right)^{T} C_{i}\right\|^{2} \\
& +\left\|\sum_{i=1}^{2} P A_{i}^{T}\left(A_{i} \varepsilon(k) B_{i}+C_{i} \varepsilon(k)^{T} D_{i}\right) B_{i}^{T} P\right\|^{2} \\
& \left.+\left\|\sum_{i=1}^{2} P D_{i}\left(A_{i} \varepsilon(k) B_{i}+C_{i} \varepsilon(k)^{T} D_{i}\right)^{T} C_{i} P\right\|^{2}\right\}
\end{aligned}
$$




$$
\begin{aligned}
\leq & \|\varepsilon(k)\|^{2}-\omega \sum_{i=1}^{2} \operatorname{tr}\left(\left(A_{i} \varepsilon(k) B_{i}+C_{i} \varepsilon(k)^{T} D_{i}\right)\right. \\
& \left.\times\left(A_{i} \varepsilon(k) B_{i}+C_{i} \varepsilon(k)^{T} D_{i}\right)^{T}\right) \\
& +\frac{\omega^{2}}{2} \sum_{i=1}^{2}\left\{\left\|A_{i}\right\|^{2}\left\|B_{i}\right\|^{2}+\left\|D_{i}\right\|^{2}\left\|C_{i}\right\|^{2}\right\}\left\|A_{i} \varepsilon(k) B_{i}+C_{i} \varepsilon(k)^{T} D_{i}\right\|^{2} \\
\leq & \|\varepsilon(k)\|^{2}-\omega\left[1-\frac{\omega}{2} \sum_{i=1}^{2}\left(\left\|A_{i}\right\|^{2}\left\|B_{i}\right\|^{2}+\left\|D_{i}\right\|^{2}\left\|C_{i}\right\|^{2}\right)\right] \\
& \times \sum_{i=1}^{2}\left\|A_{i} \varepsilon(k) B_{i}+C_{i} \varepsilon(k)^{T} D_{i}\right\|^{2} \\
\leq & \|\varepsilon(1)\|^{2}-\omega\left[1-\frac{\omega}{2} \sum_{i=1}^{2}\left(\left\|A_{i}\right\|^{2}\left\|B_{i}\right\|^{2}+\left\|D_{i}\right\|^{2}\left\|C_{i}\right\|^{2}\right)\right] \\
& \times \sum_{t=1}^{k} \sum_{i=1}^{2}\left\|A_{i} \varepsilon(t) B_{i}+C_{i} \varepsilon(t)^{T} D_{i}\right\|^{2} .
\end{aligned}
$$

From (2.14) and (2.17), it is not difficult to get

$$
\sum_{t=1}^{\infty} \sum_{i=1}^{2}\left\|A_{i} \varepsilon(t) B_{i}+C_{i} \varepsilon(t)^{T} D_{i}\right\|^{2}<\infty .
$$

The necessary condition of the series convergence (2.18) implies that

$$
\begin{gathered}
\lim _{t \rightarrow \infty}\left[A_{i} \varepsilon(t) B_{i}+C_{i} \varepsilon(t)^{T} D_{i}\right]=A_{i}\left(\lim _{t \rightarrow \infty} \varepsilon(t)\right) B_{i}+C_{i}\left(\lim _{t \rightarrow \infty} \varepsilon(t)^{T}\right) D_{i}=0, \\
\text { for } i=1,2 .
\end{gathered}
$$

By considering Lemma 2.3, we have

$$
\lim _{t \rightarrow \infty} \varepsilon(t)=0 .
$$

The proof of theorem is completed.

Similar to the proof of the above theorem, we can prove the following theorem.

Theorem 2.2. If the coupled matrix equations (1.4) have a unique anti-reflexive solution $X$, then iterative solution $X(k)$ given by Algorithm 2.2 converges to 
$X$ for any initial anti-reflexive matrix $X(1)$, if the parameter $\omega$ satisfies the inequality

$$
0<\omega<2\left[\sum_{i=1}^{2}\left(\left\|A_{i}\right\|^{2}\left\|B_{i}\right\|^{2}+\left\|C_{i}\right\|^{2}\left\|D_{i}\right\|^{2}\right)\right]^{-1} .
$$

Remark 2.1. The convergence factor in (2.14) and (2.19) may also be taken as:

$$
\begin{gathered}
0<\omega<2 \\
\times\left[\sum_{i=1}^{2}\left(\lambda_{\max }\left(A_{i} A_{i}^{T}\right) \lambda_{\max }\left(B_{i} B_{i}^{T}\right)+\lambda_{\max }\left(C_{i} C_{i}^{T}\right) \lambda_{\max }\left(D_{i} D_{i}^{T}\right)\right)\right]^{-1} .
\end{gathered}
$$

\section{Numerical examples}

In this section, we give two examples to illustrate the convergence of Algorithms 2.1 and 2.2, respectively. All the tests are performed by MATLAB.

Example 3.1. As the first example we consider the linear matrix equation $A X B+C X^{T} D=M$ with

$$
\begin{aligned}
& A=\left(\begin{array}{ccccc}
-3.7972 & 0.7621 & 0.6154 & 0.4057 & 0.0579 \\
0 & -3.2532 & 0.7919 & 0.9355 & 0.3529 \\
0 & 0 & -3.4748 & 0.9169 & 0.8132 \\
0 & 0 & 0 & -3.6205 & 0.0099 \\
0 & 0 & 0 & 0 & -3.8103
\end{array}\right) \text {, } \\
& B=\left(\begin{array}{lllll}
1 & 1 & 1 & 1 & 1 \\
1 & 1 & 1 & 1 & 1 \\
1 & 1 & 1 & 1 & 1 \\
1 & 1 & 1 & 1 & 1 \\
1 & 1 & 1 & 1 & 1
\end{array}\right) \\
& C=\left(\begin{array}{ccccc}
-4.3881 & 0.2259 & 0.2091 & 0 & 0 \\
0.4235 & -4.0637 & 0.3798 & 0.7942 & 0 \\
0.5155 & 0.7604 & -3.4846 & 0.0592 & 0.8744 \\
0.3340 & 0.5298 & 0.6808 & -4.0259 & 0.0150 \\
0.4329 & 0.6405 & 0.4611 & 0.0503 & -3.8072
\end{array}\right),
\end{aligned}
$$




$$
\begin{gathered}
D=\left(\begin{array}{ccccc}
4.7027 & -0.6979 & -0.4966 & -0.6602 & -0.7271 \\
0 & 4.9568 & -0.8998 & -0.3420 & -0.3093 \\
0 & 0 & 4.2523 & -0.2897 & -0.8385 \\
0 & 0 & 0 & 4.1991 & -0.5681 \\
0 & 0 & 0 & 0 & 4.9883
\end{array}\right), \\
M=\left(\begin{array}{ccccc}
118.0878 & -23.1868 & -53.8639 & -12.1011 & -52.2842 \\
-6.3715 & 105.8088 & -24.0695 & -58.8678 & 9.0772 \\
-64.4487 & -13.9112 & 93.5506 & 1.4371 & -69.5242 \\
13.4263 & -77.6180 & 16.4496 & 11.4427 & 16.3479 \\
-36.0898 & -0.8957 & -51.6715 & 21.7130 & 141.3938
\end{array}\right) .
\end{gathered}
$$

It can be verified that this matrix equation is consistent over reflexive matrices and has the reflexive solution

$$
X^{*}=\left(\begin{array}{ccccc}
-5.5945 & 0 & 3.2309 & 0 & 2.1158 \\
0 & -4.5064 & 0 & 3.8709 & 0 \\
2.0000 & 0 & -4.9497 & 0 & 3.6263 \\
0 & 2.0000 & 0 & -5.2410 & 0 \\
2.0000 & 0 & 2.0000 & 0 & -5.6207
\end{array}\right) \mathbb{R}_{r}^{5 \times 5}(P),
$$

with

$$
P=\left(\begin{array}{ccccc}
-1 & 0 & 0 & 0 & 0 \\
0 & 1 & 0 & 0 & 0 \\
0 & 0 & -1 & 0 & 0 \\
0 & 0 & 0 & 1 & 0 \\
0 & 0 & 0 & 0 & -1
\end{array}\right)
$$

Choose arbitrary initial iterative matrix $X(1)=0$. By Algorithm 2.1, we obtain the sequence $X(k)$. In Figure 1, we report the obtained results with several values of $\omega$ where $\delta(k)=\log _{10} \frac{\left\|X(k)-X^{*}\right\|}{\left\|X^{*}\right\|}$ and $r(k)=\log _{10}\left\|M-A X(k) B-C X(k)^{T} D\right\|$. It can be observed from Figure 1 that Algorithm 2.1 is effective. The effect of changing the convergence factor $\omega$ is illustrated in Figure 1. We see that the larger the convergence factor $\omega$ is, the faster the convergence the algorithm. 

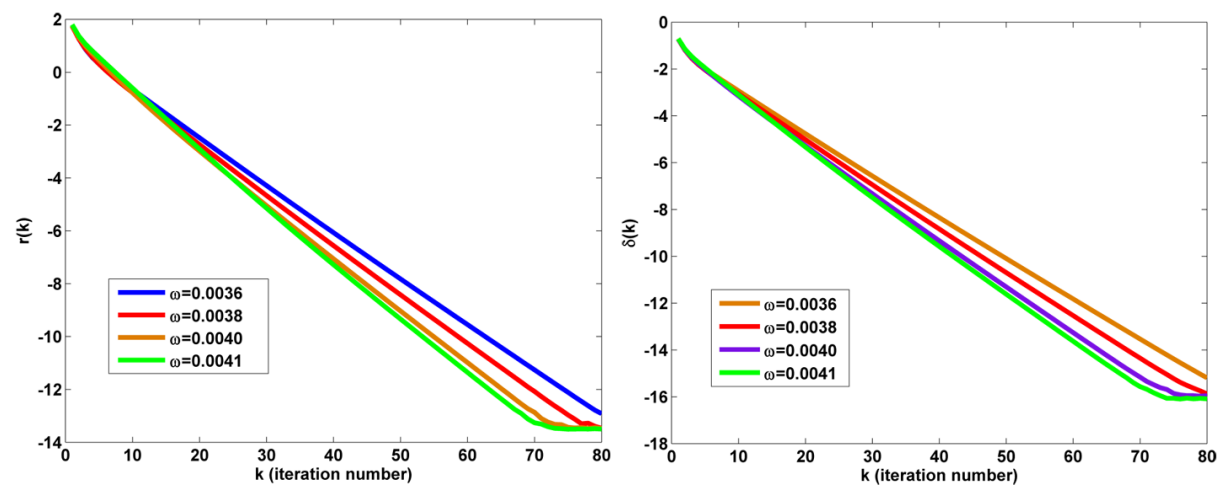

Figure 1 - The results of Example 3.1.

Example 3.2. Consider a pair of matrix equations in the form of (1.2) with the following parameters:

$$
\begin{gathered}
A_{1}=\left(\begin{array}{ccccc}
-3.7972 & 1.5242 & 1.2309 & 0.8114 & 0.1158 \\
0 & -3.2532 & 1.5839 & 1.8709 & 0.7057 \\
0 & 0 & -3.4748 & 1.8338 & 1.6263 \\
0 & 0 & 0 & -3.6205 & 0.0197 \\
0 & 0 & 0 & 0 & -3.8103
\end{array}\right), \\
B_{1}=\left(\begin{array}{ccccc}
3.6756 & 0 & 0.6085 & 0.0576 & 0.0841 \\
0 & 3.4508 & 0 & 0.3676 & 0.4544 \\
0 & 0 & 3.2324 & 0 & 0.4418 \\
0 & 0 & 0 & 3.0784 & 0 \\
0 & 0 & 0 & 0 & 3.9943
\end{array}\right) \\
A_{2}=\left(\begin{array}{ccccc}
5.5536 & 0.2259 & 0.2091 & 0 & 0 \\
0.4235 & 5.2233 & 0.3798 & 0.7942 & 0 \\
0.5155 & 0.7604 & 5.0513 & 0.0592 & 0.8744 \\
0.3340 & 0.5298 & 0.6808 & 5.2317 & 0.0150 \\
0.4329 & 0.6405 & 0.4611 & 0.0503 & 5.3431
\end{array}\right)
\end{gathered}
$$




$$
\begin{gathered}
B_{2}=\left(\begin{array}{ccccc}
-5.7027 & -0.6979 & -0.4966 & -0.6602 & -0.7271 \\
0 & -5.9568 & -0.8998 & -0.3420 & -0.3093 \\
0 & 0 & -5.2523 & -0.2897 & -0.8385 \\
0 & 0 & 0 & -5.1991 & -0.5681 \\
0 & 0 & 0 & 0 & -5.9883
\end{array}\right), \\
C_{1}=\left(\begin{array}{ccccc}
17.1697 & -56.8568 & 33.5482 & -25.9479 & 24.4829 \\
-10.1615 & 15.8018 & -43.9289 & 33.5031 & -33.0153 \\
13.4809 & -12.7575 & 14.0869 & -51.7608 & 15.1875 \\
-26.6155 & 0.1361 & -27.8120 & -0.2810 & -33.2835 \\
0 & -26.2971 & 0 & -26.2605 & -3.4625
\end{array}\right),
\end{gathered}
$$

and

$$
C_{2}=\left(\begin{array}{ccccc}
-2.5771 & -169.8176 & -31.9603 & -121.1339 & -26.8271 \\
-68.6326 & -25.6598 & -158.7006 & -36.3862 & -152.1132 \\
-9.3473 & -87.2429 & -35.0790 & -174.8441 & -43.6301 \\
-65.7124 & -26.3748 & -77.8285 & -38.9935 & -98.0133 \\
-7.8789 & -83.1321 & -31.0110 & -83.9174 & -30.2760
\end{array}\right) .
$$

We can verify the pair of matrix equations in the form of (1.2) are consistent over anti-reflexive matrix $X$ and have the anti-reflexive solution

$$
X^{*}=\left(\begin{array}{ccccc}
0 & 5.0484 & 0 & 3.6228 & 0 \\
2.0000 & 0 & 5.1677 & 0 & 3.4115 \\
0 & 2.0000 & 0 & 5.6676 & 0 \\
2.0000 & 0 & 2.0000 & 0 & 2.0394 \\
0 & 2.0000 & 0 & 2.0000 & 0
\end{array}\right) \mathbb{R}_{a}^{5 \times 5}(P) .
$$

Taking $X(1)=0$, we apply Algorithm 2.2 to compute $X(k)$. The effect of changing the convergence factor $\omega$ is illustrated in Figure 2 where

$$
\delta(k)=\log _{10} \frac{\left\|X(k)-X^{*}\right\|}{\left\|X^{*}\right\|} \text { and } r(k)=\log _{10} \sum_{i=1}^{2}\left\|C_{i}-A_{i} X(k) B_{i}\right\| .
$$

Obviously both $\delta(k)$ and $r(k)$ decrease, and converge to zero as $k$ increases. 

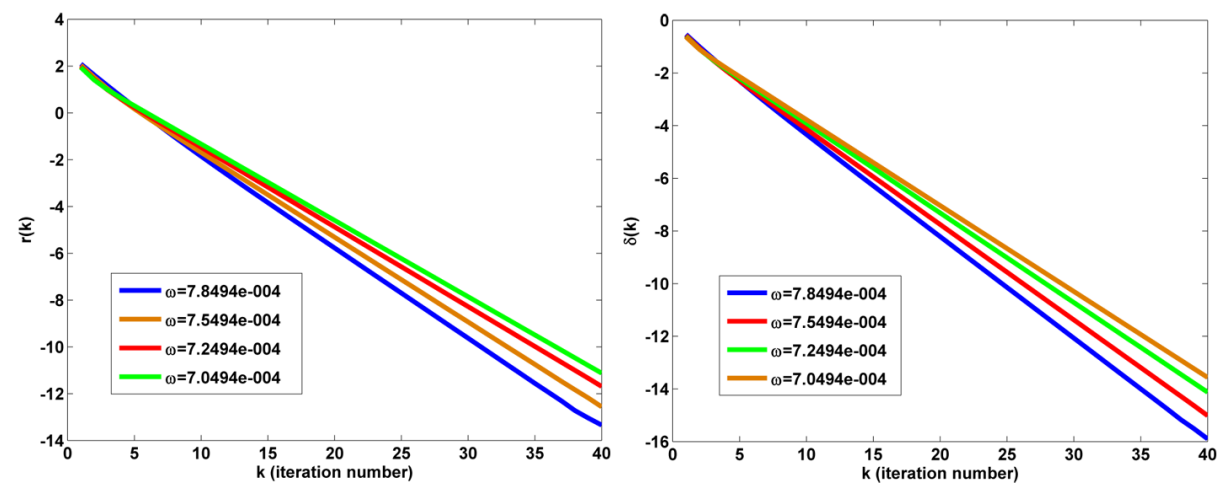

Figure 2 - The results of Example 3.2.

\section{Concluding remarks}

In this paper, we have considered the coupled matrix equations (1.4) over reflexive and anti-reflexive matrices. First Algorithms 2.1 and 2.2 were introduced for finding reflexive and anti-reflexive solutions of (1.4). Second the convergence theorems of the iterative algorithms were presented. The experiments are encouraging and seem to indicate that Algorithms 2.1 and 2.2 work well for numerical examples. It is interesting to develop the introduced algorithms for solving other linear matrix equations. We leave it as a topic for further research.

Acknowledgements. The authors are very much indebted to an anonymous referee for his/her valuable comments and careful reading of the manuscript.

\section{REFERENCES}

[1] A. Andrew, Eigenvectors of certain matrices. Linear Algebra Appl., 7 (1973), $157-162 ; 455-460$.

[2] A. Ben-Israel, A Cramer rule for least-norm solutions of consistent linear equations. Linear Algebra Appl., 43 (1982), 223-226.

[3] H.C. Chen, Generalized reflexive matrices: special properties and applications. SIAM J. Matrix Anal. Appl., 19 (1998), 140-153.

[4] L. Dai, Singular Control Systems, Berlin: Springer-Vertag (1989).

[5] H. Dai, On the symmetric solutions of linear matrix equations. Linear Algebra Appl., 131 (1990), 1-7. 
[6] L. Datta and S. Morgera, Some results on matrix symmetries and a pattern recognition application. IEEE Trans. Signal Process, 34 (1986), 992-994.

[7] L. Datta and S. Morgera, On the reducibility of centrosymmetric matricesapplications in engineering problems. Circuits Systems Signal Process, 8 (1989), 71-96.

[8] M. Dehghan and M. Hajarian, The general coupled matrix equations over generalized bisymmetric matrices. Linear Algebra Appl., 432 (2010), 1531-1552.

[9] M. Dehghan and M. Hajarian, The reflexive and anti-reflexive solutions of a linear matrix equation and systems of matrix equations. Rocky Mountain J. Math., 40 (2010), 1-23.

[10] M. Dehghan and M. Hajarian, On the generalized bisymmetric and skew-symmetric solutions of the system of generalized Sylvester matrix equations. Linear and Multilinear Algebra, 59 (2011), 1281-1309.

[11] M. Hajarian and M. Dehghan, The generalized centro-symmetric and least squares generalized centro-symmetric solutions of the matrix equation $A Y B+C Y^{T} D=$ E. Mathematical Methods in the Applied Sciences, 34 (2011), 1562-1579.

[12] M. Dehghan and M. Hajarian, Solving the generalized Sylvester matrix equation $\sum_{i=1}^{p} A_{i} X B_{i}+\sum_{j=1}^{q} C_{j} Y D_{j}=E$ over reflexive and anti-reflexive matrices. International Journal of Control, Automation and Systems, 9 (2011), 118-124.

[13] M. Dehghan and M. Hajarian, Analysis of an iterative algorithm to solve the generalized coupled Sylvester matrix equations. Applied Mathematical Modelling, 35 (2011), 3285-3300.

[14] M. Dehghan and M. Hajarian, Two algorithms for the Hermitian reflexive and skew-Hermitian solutions of Sylvester matrix equations. Applied Mathematics Letters, 24 (2011), 444-449.

[15] M. Dehghan and M. Hajarian, The $(R, S)$-symmetric and $(R, S)$-skew symmetric solutions of the pair of matrix equations $A_{1} X B_{1}=C_{1}$ and $A_{2} X B_{2}=C_{2}$. Bulletin of the Iranian Mathematical Society, 37 (2011), 269-279.

[16] M. Dehghan and M. Hajarian, SSHI methods for solving general linear matrix equations. Engineering Computations, 28 (2012), 1028-1043.

[17] M. Dehghan and M. Hajarian, The generalised Sylvester matrix equations over the generalised bisymmetric and skew-symmetric matrices. International Journal of Systems Science, 43 (2012), 1580-1590. 
[18] M. Dehghan and M. Hajarian, Iterative algorithms for the generalized centrosymmetric and central anti-symmetric solutions of general coupled matrix equations. Engineering Computations, 29 (2012), 528-560.

[19] J. Delmas, On Adaptive EVD asymptotic distribution of centro-symmetric covariance matrices. IEEE Trans. Signal Process, 47 (1999), 1402-1406.

[20] Z.J. Bai, The inverse eigenproblem of centrosymmetric matrices with a submatrix constraint and its approximation. SIAM J. Matrix Anal. Appl., 26 (2005), 11001114.

[21] P. Delsarte and Y. Genin, Spectral properties of finite Toeplitz matrices, in Proceedings of the 1983 International Symposium on Mathematical Theory of Networks and Systems, Beer Sheva, Israel, 1983, Springer-Verlag, Berlin, New York (1984), 194-213.

[22] F. Ding and T. Chen, Gradient based iterative algorithms for solving a class of matrix equations. IEEE Trans. Autom. Contr., 50 (2005), 1216-1221.

[23] F. Ding and T. Chen, Hierarchical gradient-based identification of multivariable discrete-time systems. Automatica, 41 (2005), 315-325.

[24] F. Ding and T. Chen, Iterative least squares solutions of coupled Sylvester matrix equations. Systems Control Lett., 54 (2005), 95-107.

[25] F. Ding and T. Chen, Hierarchical least squares identification methods for multivariable systems. IEEE Trans. Autom. Contr., 50 (2005), 397-402.

[26] F. Ding and T. Chen, On iterative solutions of general coupled matrix equations. SIAM J. Control Optim., 44 (2006), 2269-2284.

[27] F. Ding, P.X. Liu and J. Ding, Iterative solutions of the generalized Sylvester matrix equations by using the hierarchical identification principle. Appl. Math. Comput., 197 (2008), 41-50.

[28] G.R. Duan, The solution to the matrix equation $A V+B W=E V J+R$. Appl. Math. Lett., 17 (2004), 1197-1202.

[29] L.R. Fletcher, J. Kuatsky and N.K. Nichols, Eigenstructure assignment in descriptor systems. IEEE Trans. Auto. Contr., 31 (1986), 1138-1141.

[30] P.M. Frank, Fault diagnosis in dynamic systems using analytical and knowledgebased redundancy-a survey and some new results. Automatica, 26 (1990), 459474.

[31] J. Gilbert and L. Gilbert, Linear Algebra and Matrix Theory, $2^{\text {nd }}$ edition. Thomson Brooks/Cole (2005). 
[32] G.H. Golub and C.F. Van Loan, Matrix computations, third ed., The Johns Hopkins University Press, Baltimore and London (1996).

[33] R.A. Horn and C.R. Johnson, Topics in Matrix Analysis, Cambridge University Press (1991), 259-260.

[34] A.B. Israel and T.N.E. Greville, Generalized inverses theory and applications, $2^{\text {nd }}$ ed., Springer-Verlag, New York (2003).

[35] I.I. Kyrchei, Cramer's rule for quaternionic systems of linear equations. Journal of Mathematical Sciences, 155 (2008), 839-858.

[36] I.I. Kyrchei, Analogs of the adjoint matrix for generalized inverses and corresponding Cramer rules. Linear and Multilinear Algebra, 56 (2008), 453-46.

[37] I.I. Kyrchei, Cramer's rule for some quaternion matrix equations next term. Appl. Math. Comput., 217 (2010), 2024-2030.

[38] S.K. Mitra, Common solutions to a pair of linear matrix equations $A_{1} X B_{1}=$ $C_{1}, A_{2} X B_{2}=C_{2}$. Proc. Camb. Philos. Soc., 74 (1973), 213-216.

[39] A. Navarra, P.L. Odell and D.M. Young, A representation of the general common solution to the matrix equations $A_{1} X B_{1}=C_{1}$ and $A_{2} X B_{2}=C_{2}$ with applications. Comput. Math. Appl., 41 (2001), 929-935.

[40] F. Piao, Q. Zhang and Z. Wang, The solution to matrix equation $A X+X^{T} C=B$. J. Franklin Institute, 344 (2007), 1056-1062.

[41] M. Wang, X. Cheng and M. Wei, Iterative algorithms for solving the matrix equation $A X B+C X^{T} D=E$. Appl. Math. Comput., 187 (2007), 622-629.

[42] J. Weaver, Centrosymmetric (cross-symmetric) matrices, their basic properties, eigenvalues, and eigenvectors. Amer. Math. Monthly, 92 (1985), 711-717.

[43] F.Z. Zhou, X.Y. Hu and L. Zhang, The solvability conditions for the inverse eigenvalue problem of generalized centro-symmetric matrices. Linear Algebra Appl., 364 (2003), 147-160.

[44] B. Zhou and G.R. Duan, A new solution to the generalized Sylvester matrix equation $A V-E V F=B W$. Systems Control Lett., 55 (2006), 193-198.

[45] B. Zhou and G.R. Duan, Solutions to generalized Sylvester matrix equation by Schur decomposition. Internat. J. Systems Sci., 38 (2007), 369-375.

[46] B. Zhou, Z.Y. Li, G.R. Duan and Y. Wang, Weighted least squares solutions to general coupled Sylvester matrix equations. J. Comput. Appl. Math., 224 (2009), 759-776. 